\title{
If Theme: Neurology and Development
}

\author{
Established status epilepticus treatment trial (ESTT) \\ (Lancet. 2020;395:1217-24)
}

Currently, there is a lack of good quality evidence for the second line anti-seizure medications in the management of status epilepticus. The researchers of this multi-center, double-blind, response-adaptive, randomized controlled trial, done in 58 hospital emergency departments across the USA enrolled 462 patients (224 aged 2-18 years). Patients were eligible for inclusion if they had been treated for a generalized convulsive seizure of longer than 5-min duration with adequate doses of benzodiazepines, and continued to have persistent or recurrent convulsions in the emergency department for at least 5-min and no more than $30 \mathrm{~min}$ after the last dose of benzodiazepine. The primary outcome of absence of clinically apparent seizures with improved consciousness and without additional anti-seizure medication at 1-h from start of drug infusion was achieved in 52\% (95\% CI 41-62\%) of children receiving levetiracetam, 49\% (38-61\%) receiving fosphenytoin, and 52\% (41-63\%) getting valproate. No differences were detected in efficacy or primary safety outcome (life-threatening hypotension or cardiac arrhythmia). Endotracheal intubation of children occurred more frequently in the fosphenytoin group (33\%)than in levetiracetam group (8\%) or valproate group (11\%). Any of the three drugs can be considered as a potential firstchoice, second-line drug for benzodiazepine-refractory status epilepticus.

Levetiracetamversus phenobarbital for neonatal seizures (NEOLEV2) (Pediatrics. 2020;145:e20193182)

Phenobarbital and phenytoin frequently fail to control neonatal seizures and there are concerns about the safety of these anti-seizure medications in the developing brain. Levetiracetamis increasingly being used in neonates without good quality evidence supporting its use. The researchers in this multicenter, randomized, blinded, controlled, phase IIb trial to study the efficacy and safety of levetiracetam $(40 \mathrm{mg} / \mathrm{kg})$ compared with phenobarbital $(20 \mathrm{mg} / \mathrm{kg})$ as a first-line treatment for neonatal seizures of any cause,analyzed 83 neonates who met the study criteria (53 received levetiracetam and 30 phenobarbitone). The primary outcome measure was complete seizure freedom for 24 hours, assessed by independent review of the EEGs by two neurophysiologists.In the phenobarbital group $80 \%$ patients remained seizure free for 24 hours, compared with $28 \%$ of patients inlevetiracetam $(P=0.001)$. There appears to be a need for studies with higher doses of levetiracetam and with longer follow-up for adverse effects.

Drugs for acute attack of pediatric migraine (Clin Neurol Neurosurg.2020;195:105853)

To know the efficacy of the various drugs for the acute management of migraine in children, a network metaanalysis of high quality trials (6029 migraineurs) randomly assigned to 14 different drugs was done. The outcome measure used was pain-freedom and pain relief at 2 hours post-dose. Sumatriptan nasal spray and zolmitriptan nasal spray were superior to placebo in the two efficacy outcomes, whereas almotriptan, rizatriptan, sumatriptan with naproxen sodium, and ibuprofen were superior to placebo only in one of the efficacy outcomes. In network meta-analysis, the best three treatments for achieving freedom from pain were ibuprofen, sumatriptan with naproxen sodium and ibuprofen suspension. Meanwhile, the best three treatments for pain relief at 2 hours were ibuprofen suspension, ibuprofen, and rizatriptan. In conclusion, in acute treatments of pediatric migraine, most triptans and NSAIDs were effective to achieve painfreedom or pain-relief.

Prednisolone in autistic spectrum disorder $(\mathrm{J}$ Pediatr (Rio J). 2020:S0021-7557(19)30465-6)

Autistic spectrum disorder may have an inflammatory or autoimmune etiology as supported by increased risk of ASD in children with a maternal history of rheumatoid arthritis or celiac disease. To study the role of immunomodulation using steroids, the authors designed a prospective, double-blinded, randomized, placebocontrolled clinical trial. Thirty eight boys aged between 3 to 7 years out of 40 enrolled ( 20 were randomized to the placebo group and 18 to the steroid group receiving prednisolone for 24 weeks, at an initial dose of $1 \mathrm{mg} / \mathrm{kg}$ / day and a tapering dose from the ninth week onwards) completed the study. Twenty out of 38 had history of language regression. Language was measured on four occasions over a 12-month period using standardized scales. Though there was no significant change in language score in both groups, there was change in the language scores in younger children and in those with language regression. More studies are needed to address the role of immunomodulation in children with autistic spectrum disorder.

MAHESh KaMATE drmaheshkamate@gmail.com 